Virginia Commonwealth University VCU Scholars Compass

2009

\title{
Magnetic response of core-shell cobalt ferrite nanoparticles at low temperature
}

K. Maaz

PINSTECH

M. Usman

Quaid-i-Azam University

S. Karim

PINSTECH

See next page for additional authors

Follow this and additional works at: http://scholarscompass.vcu.edu/phys_pubs

Part of the Physics Commons

Maaz, K., Usman, M., \& Karim, S., et al. Magnetic response of core-shell cobalt ferrite nanoparticles at low temperature. Journal of Applied Physics, 105, 113917 (2009). Copyright (C) 2009 American Institute of Physics.

\section{Downloaded from}

http://scholarscompass.vcu.edu/phys_pubs/122

This Article is brought to you for free and open access by the Dept. of Physics at VCU Scholars Compass. It has been accepted for inclusion in Physics Publications by an authorized administrator of VCU Scholars Compass. For more information, please contact libcompass@vcu.edu. 
Authors

K. Maaz, M. Usman, S. Karim, A. Mumtaz, S. K. Hasanain, and M. F. Bertino 


\title{
Magnetic response of core-shell cobalt ferrite nanoparticles at low temperature
}

\author{
K. Maaz, ${ }^{1, a)}$ M. Usman, ${ }^{2}$ S. Karim, ${ }^{1}$ A. Mumtaz, ${ }^{2}$ S. K. Hasanain, ${ }^{2}$ and M. F. Bertino ${ }^{3}$ \\ ${ }^{1}$ Physics Division, PINSTECH, Post Office Nilore, Islamabad, Pakistan \\ ${ }^{2}$ Department of Physics, Quaid-i-Azam University, Islamabad, Pakistan \\ ${ }^{3}$ Department of Physics, Virginia Commonwealth University, Richmond, Virginia 23824, USA
}

(Received 3 March 2009; accepted 25 April 2009; published online 5 June 2009)

\begin{abstract}
Cobalt ferrite nanoparticles (size: $26 \pm 4 \mathrm{~nm}$ ) have been synthesized by coprecipitation route. The coercivity of nanoparticles follows a simple model of thermal activation of particle moments over the anisotropy barrier in the temperature range of 30-300 K in accordance with Kneller's law; however, at low temperatures $(<30 \mathrm{~K})$, the coercivity shows some deviation from this law. The saturation magnetization follows the modified Bloch's law in the temperature range of 10-300 K. Exchange bias $\left(H_{\mathrm{ex}}\right)$ studies of the samples show that $H_{\mathrm{ex}}$ increases with decreasing temperature of the sample. A strong increase in the $H_{\text {ex }}$ values is found below $30 \mathrm{~K}$ for the low applied field $( \pm 20 \mathrm{kOe})$, while a smaller increase is found for the high applied field $( \pm 90 \mathrm{kOe})$. The slow increase in the exchange bias at high applied field has been attributed to the high field effects on the surface (shell) spins. These shell spins align along the field direction that weakens the core-shell interface interactions leading to the reduction in the exchange bias at lower temperatures. (C) 2009 American Institute of Physics. [DOI: 10.1063/1.3139293]
\end{abstract}

\section{INTRODUCTION}

Cobalt ferrite $\left(\mathrm{CoFe}_{2} \mathrm{O}_{4}\right)$ is a well-known hard magnetic material with a relatively high coercivity, exchange bias, and moderate magnetization. These properties, along with its chemical and physical stability, make cobalt ferrite very promising candidate for a variety of applications in computer, electronics, and biomedical industries. ${ }^{1-4} \mathrm{CoFe}_{2} \mathrm{O}_{4}$ in its bulk form has the inverse spinel structure where $\mathrm{Fe}^{+2}$ ions are equally filled in tetrahedral and octahedral sites, while $\mathrm{Co}^{+2}$ ions are filled in octahedral sites only. Magnetic properties of $\mathrm{CoFe}_{2} \mathrm{O}_{4}$ depend on the type of the cations and their distribution among the tetrahedral and octahedral sites. It has been observed that the resulting magnetic properties of nanocrystalline $\mathrm{CoFe}_{2} \mathrm{O}_{4}$ are entirely different from its bulk counterpart, which makes this material of great scientific and technological interests. ${ }^{5-8}$ The bulk cobalt ferrite has coercivity, saturation magnetization, and Curie's temperature of $900 \mathrm{Oe}, 80 \mathrm{emu} / \mathrm{g}$, and $790 \mathrm{~K}$, respectively, which are considerably different from its nanocrystalline form. ${ }^{9-11}$ There is a considerable interest in the magnetism and related phenomena in nanoscale $\mathrm{CoFe}_{2} \mathrm{O}_{4}$. The complexity of the situation stems from several competing effects that are sometimes difficult to isolate in these nanoparticles. These include the surface effects, finite size effects, and interparticle interactions. It is now well established that in small size particles, on nanoscale, the surface spins play a dominant role as far as the magnetic properties in these particles are concerned. These spins cause, for example, the reduction in saturation magnetization and enhancement in coercivity with decreasing particle sizes at the nanometric range. Kodama and

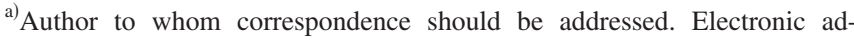
dresses: maaz@impcas.ac.cn and maaz@phys.qau.edu.pk. Tel.: +92-3215029820. FAX: +92-51-9290275.
}

Berkowitz $^{12,13}$ showed that in ferrite nanoparticles, due to the broken symmetries and exchange bonds at the surfaces, the spins at the surface of ferrite nanoparticles do not follow the core anisotropy direction and become disordered or may become canted leading to even high anisotropy compared to the core of the nanoparticles. Such particles are generally termed as the core-shell nanoparticles, where the core spins behave like ferromagnetic (FM) or ferrimagnetic (FI), and the shell is composed of disordered spins or canted spins. The coreshell picture is not only limited to the ferrite nanoparticles, this also includes other configurations such as $\mathrm{CoO}, \mathrm{FeO}$, and other complex oxides as well.

In this work, we report low temperature magnetic properties of $26 \pm 4 \mathrm{~nm}$ cobalt ferrite nanoparticles and the results are explained with reference to the disordered surface spins, finite size effects, and thermal activation model. This has not been reported in detail previously, to the best of our knowledge. One of the interesting phenomena observed by the core-shell nanoparticles is the exchange bias effect, i.e., when the particles are cooled in the presence of some magnetic field, the resultant hysteresis loop appears shifted (asymmetric) along both the field and magnetization axes. The appearance of the shifted hysteresis loop is generally attributed to the core-shell nature of the nanoparticles. Here the exchange bias for these nanoparticles has also been investigated from room temperature $(300 \mathrm{~K})$ to $10 \mathrm{~K}$ and the results have been explained with reference to the core-shell structure in these nanoparticles.

\section{EXPERIMENTAL}

The samples reported in this study were synthesized by coprecipitation technique that has been described in detail elsewhere. ${ }^{14,15}$ The starting materials were iron chloride, cobalt chloride, and nickel chloride, while the oleic acid was 


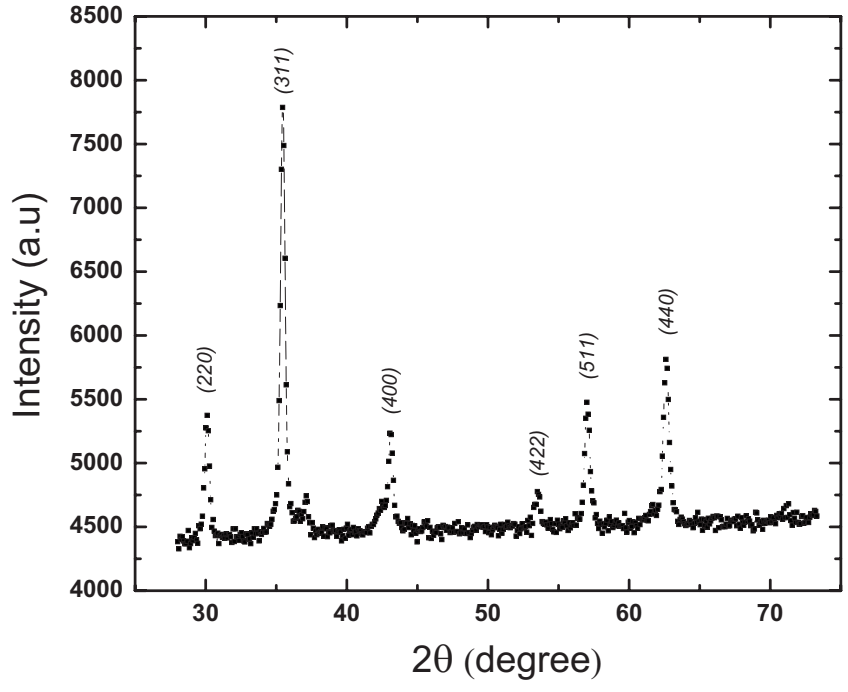

FIG. 1. XRD pattern of $\mathrm{CoFe}_{2} \mathrm{O}_{4}$ nanoparticles prepared by coprecipitation method after annealing at $600{ }^{\circ} \mathrm{C}$ with average crystallite size of about $26 \pm 4 \mathrm{~nm}$.

used as the surfactant. The reaction was carried out at $80{ }^{\circ} \mathrm{C}$ and double distilled de-ionized water was used as the solvent to minimize the impurities in the final product. After completion of the reaction, the solution was cooled to room temperature and then centrifuged in order to isolate the supernatants. The final product was dried at $100^{\circ} \mathrm{C}$ overnight and then annealed at $600{ }^{\circ} \mathrm{C}$ to get the desired particles. The size and size distribution were controlled by controlling the relative nucleation and growth rates during the reaction. Smaller and uniformly distributed particles were obtained if the nucleation rate was higher than the growth rate. Large $p \mathrm{H}$ values in the range of 12-13 were used in accordance with the prediction that higher production yields are expected for larger $p \mathrm{H}$ values. ${ }^{14}$

The physical characterization of the nanoparticles was performed by x-ray diffractometer (XRD-7000, Shimadzu, $\mathrm{Cu} K \alpha=0.154178 \mathrm{~nm}$ ) and transmission electron microscope (TEM, JEM-3010, JEOL). For the magnetic characterization of the sample, the powdered sample was mixed in a nanomagnetic epoxy raisin and was dried in a cylindrical mold (height of $4 \mathrm{~mm}$ and diameter of $2 \mathrm{~mm}$ ). The dc magnetic measurements were performed by quantum design physical property measurement system.

\section{RESULTS AND DISCUSSION}

The x-ray diffraction (XRD) pattern of cobalt ferrite nanoparticles is shown in Fig. 1. All the peaks in the pattern correspond to the expected inverse spinel structure of $\mathrm{CoFe}_{2} \mathrm{O}_{4}$. These peaks are indexed to the cubic $\mathrm{CoFe}_{2} \mathrm{O}_{4}$ phase according to Ref. 16. The particle size has been calculated from the XRD line broadening of the (311) peak using Scherrer's equation. ${ }^{17}$ Figure 2 shows the transmission electron microscopy image of the prepared nanoparticles. In the transmission electron microscopy (TEM) analysis we see that most of the particles appear spherical in shape, separated from each other; however, some agglomerated particles are also present in the sample that appear like a single big particle in the image. The inset in Fig. 2 shows the selected area

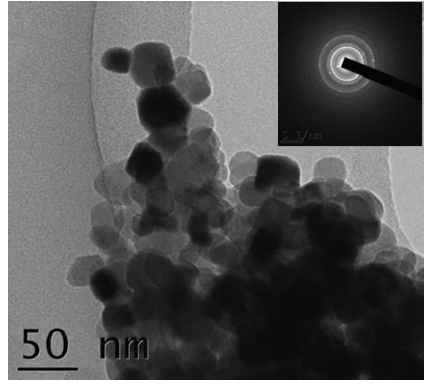

FIG. 2. High resolution TEM of $\mathrm{CoFe}_{2} \mathrm{O}_{4}$ nanoparticles. The inset in the figure shows the SAED analysis of the sample.

electron diffraction (SAED) pattern of the sample that represents the well crystalline nature of the prepared nanoparticles. It is to be noted that the size of the nanoparticles as calculated from the XRD pattern has been found in good matching with TEM visual results.

The magnetization measurements were made at different temperatures, starting from room temperature $(300 \mathrm{~K})$ down to $10 \mathrm{~K}$. In Fig. 3 we have presented $M(H)$ loops at different temperatures of $10,50,100,150,200,250$, and $300 \mathrm{~K}$ for the applied fields of up to $90 \mathrm{kOe}$. The inset in the figure shows the expanded region around the origin (in the field region of $\pm 2 \mathrm{kOe}$ ) in order to make the coercivity more visible at various temperatures. One of the remarkable features in the $M(H)$ loops is that the magnetization does not saturate, especially at low temperatures, even at high applied field of $90 \mathrm{kOe}$ (see Fig. 3). This is generally attributed to the canted or disordered spins at the surface of the nanoparticles that are difficult to align along the field direction, causing an unsaturated magnetization in these nanoparticles at low temperatures. The coercivity values at different temperatures, as calculated from the $M(H)$ loops, are plotted in Fig. 4. We observed a monotonic increase in coercivity with decreasing temperature of the nanoparticles. The reason for the increasing coercivity with decreasing temperature can be under-

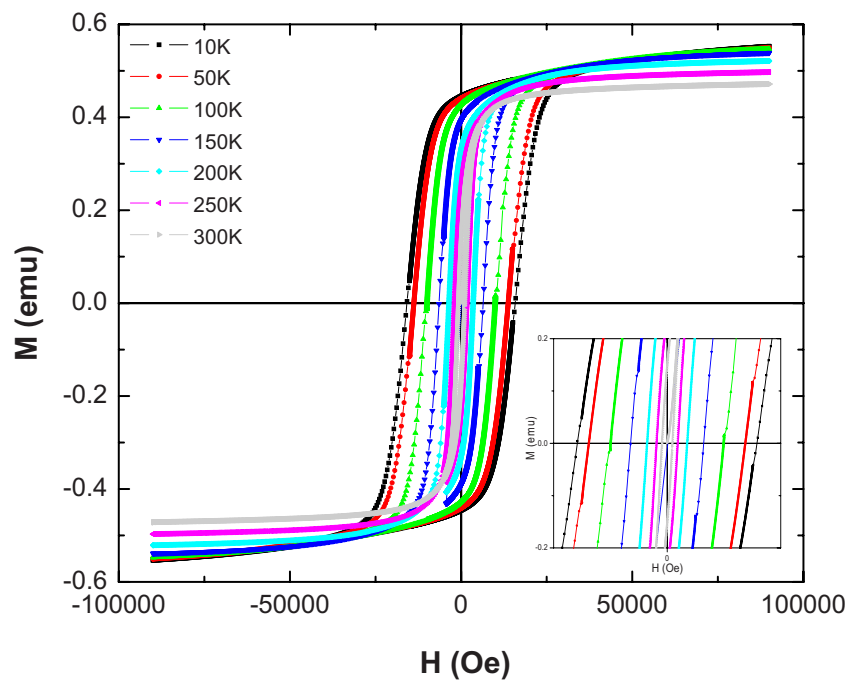

FIG. 3. (Color online) $M(H)$ loops of $\mathrm{CoFe}_{2} \mathrm{O}_{4}$ nanoparticles taken at 10, $50,100,150,200,250$, and $300 \mathrm{~K}$ up to maximum field of $\pm 90 \mathrm{kOe}$. The inset in the figure shows the expanded region around the origin in order to make the coercivity visible at various temperatures. 


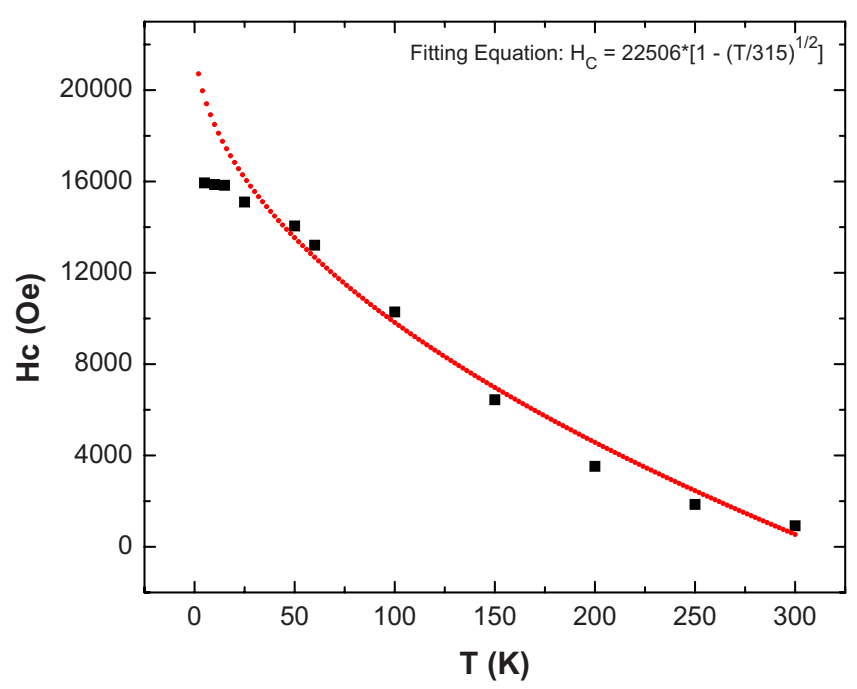

FIG. 4. (Color online) Temperature dependence of coercivity for $\mathrm{CoFe}_{2} \mathrm{O}_{4}$ nanoparticles. The curve shows the fit according to Kneller's law $\left(H_{C}\right.$ $\left.=H_{C 0}\left[1-\left(T / T_{B}\right)^{1 / 2}\right]\right)$ for our system.

stood by considering the effects of thermal fluctuations of the blocked moment, across the anisotropy barrier. For an assembly of noninteracting three-dimensional single domain magnetic nanoparticles with uniaxial anisotropy, the coercivity $\left(H_{C}\right)$ in the temperature range $\left(0-T_{B}\right)$ can be written in the form of simple model of thermal activation of particle moments over the anisotropy barriers (Kneller's law) as ${ }^{18,19}$

$$
H_{C}=H_{C_{0}}\left(1-\frac{T}{T_{B}}\right)^{1 / 2},
$$

where, $H_{C_{0}}$ is the coercivity at $T=0 \mathrm{~K}$ and can be estimated by extrapolating the $H_{C}$ versus temperature curve toward the field axis, while $T_{B}$ is the superparamagnetic blocking temperature of the nanoparticles. Referring to Fig. 4, the red line shows the fit curve according to the above relation (Kneller's law) using the blocking temperature as the fitting parameter. As evident from the figure, the data fit well to the above relation in the high temperature region (300-30 K). The coercivity at $T=0 \mathrm{~K}\left(H_{0 \mathrm{~K}}\right)$ for these nanoparticles, as determined from the fit, is $\sim 22.5 \mathrm{kOe}$, while the blocking temperature is $\sim 315 \mathrm{~K}$. Previously we have reported the coercivity and blocking temperature $\left(T_{B}\right)$ for the same sample to be $\sim 16 \mathrm{kOe}$ and $\sim 268 \mathrm{~K}$. ${ }^{14}$ The $T_{B}$ value found here $(315 \mathrm{~K})$ is larger than the value reported in Ref. 14 . The difference between the two values arises due to the fact that in our previous work the data were taken under a cooling field of $5 \mathrm{kOe}$ that usually shifts the peak to the lower values in the $M(T)$ curves. We see that Kneller's relation fits very well in the temperature range of $(300-30 \mathrm{~K})$; however, the experimental data deviate from this law (fit curve) below $\sim 30 \mathrm{~K}$. Thus the simple model of thermal activation of particle moments over the anisotropy barriers does not appear to be applicable below $30 \mathrm{~K}$ in this case. The deviation of $H_{C}$ from the thermal activation model at low temperatures can be explained in the way that for $\mathrm{CoFe}_{2} \mathrm{O}_{4}$ nanoparticles the surface spins (shell spins) freeze in their random sates, giving no further response to the applied field that resultantly prevent the core spins to further align along the field direc-

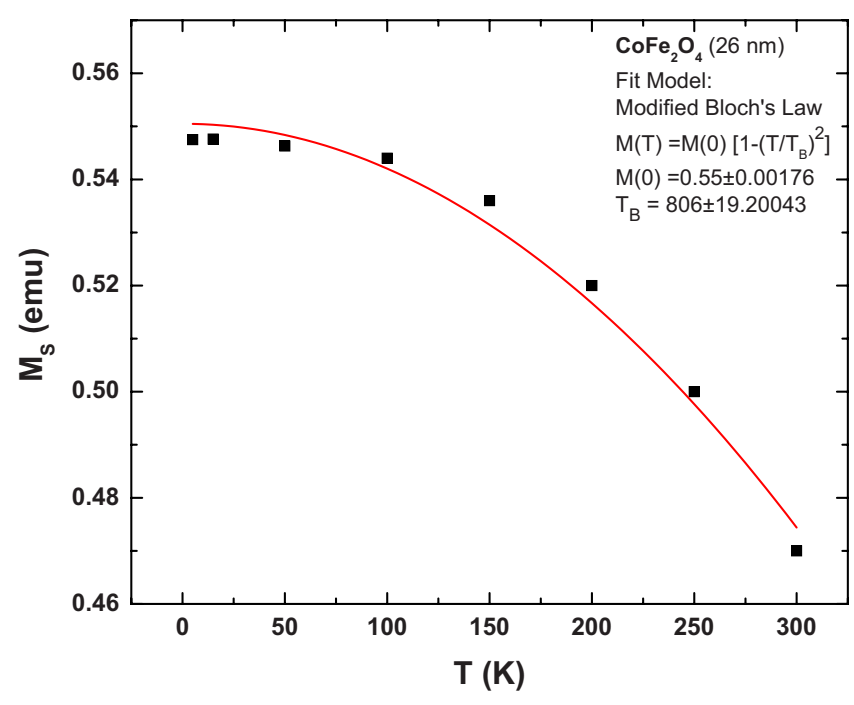

FIG. 5. (Color online) Saturation magnetization $\left(M_{S}\right)$ as a function of temperature for $\mathrm{CoFe}_{2} \mathrm{O}_{4}$ nanoparticles. The line is the fit curve according to modified Bloch's law for saturation magnetization of FM materials.

tion. This, in turn, saturates the coercive fields at low temperatures (below $30 \mathrm{~K}$ ). Our results are in agreement with the work of Iglesias and Labarta ${ }^{20}$ based on the Monte Carlo simulation of the dependence of coercivity on temperature of $\gamma-\mathrm{Fe}_{3} \mathrm{O}_{4}$ spherical nanoparticles.

The magnetization measurements at different temperatures for cobalt ferrite nanoparticles are presented in Fig. 5. From the figure, it is evident that the saturation magnetization $\left(M_{S}\right)$ increases with decreasing temperature of the sample. For a bulk FM/MI system, the saturation magnetization below the Curie temperature of the system follows Bloch's law of the form $\left[M(T)=M(0)\left[1-\left(T / T_{0}\right)^{\alpha}\right] .^{21}\right.$ Where $\left(1 / T_{0}\right)^{\alpha}$ is called the Bloch constant $(B)$ and it depends on the structure of the material. $M(T)$ is the temperaturedependent magnetization and $\alpha$ is Bloch's exponent with a value of $3 / 2$ for bulk materials. This law is generally valid for bulk material in the high temperature range. However, at the nanoscale, due the finite size effects, the thermal dependence of magnetization deviates from Bloch's law as the magnons with wavelength larger than the particle dimensions cannot be excited and a threshold of thermal energy is required to generate spin waves in these small scaled particles. Thus for nanoparticles, the spin-wave structure is modified in the form of a power law $\left(T^{\alpha}\right)$ with Bloch's exponent larger than its bulk value of $3 / 2$. This is known as the modified Bloch's law. ${ }^{22,23}$ The value of $\alpha$ in the modified Bloch's law has been experimentally determined by Auino et al. ${ }^{23}$ They found that for large sized $\mathrm{CuFe}_{2} \mathrm{O}_{4}$ nanoparticles, the value of $\alpha$ was $3 / 2$, whereas for smaller particles, this value was close to 2. The difference was attributed to finite size effects in smaller particles. Now referring back to our system of $\mathrm{CoFe}_{2} \mathrm{O}_{4}$ nanoparticles (see Fig. 5) we see that the modified Bloch's law with $\alpha=2$ (shown by the red line) is satisfied for these particles in the full temperature range of $10-300 \mathrm{~K}$. Thus the modified spin-wave spectrum is applicable to $\mathrm{CoFe}_{2} \mathrm{O}_{4}$ nanoparticles.

We now turn to the exchange bias effects in cobalt ferrite nanoparticles. Figure 6 shows the dependence of exchange 


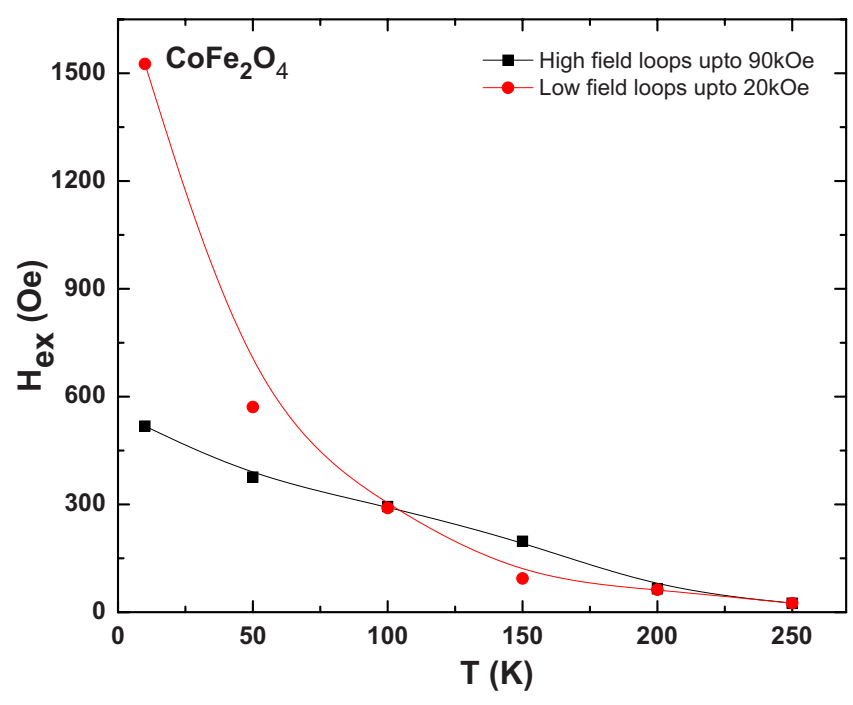

FIG. 6. (Color online) The dependence of exchange field $\left(H_{\mathrm{ex}}\right)$ on temperature for $\mathrm{CoFe}_{2} \mathrm{O}_{4}$ sample measured at different applied fields (20 and $90 \mathrm{kOe})$. The pronounced increase in $H_{\mathrm{ex}}$ values at $20 \mathrm{kOe}$ is evident at low temperatures.

field $\left(H_{\mathrm{ex}}\right)$ on temperature on the basis of two different applied fields. In the first case the loop was recorded under $\pm 90 \mathrm{kOe}$ (shown by the black line in the figure), while in the second case $M(H)$ loops were obtained under a field of $\pm 20 \mathrm{kOe}$ (shown by the red line in the figure). In both cases the cooling field was $4 \mathrm{kOe}$. It is evident from the figure that the samples exposed to $20 \mathrm{kOe}$ show more exchange bias as compared to those exposed to $90 \mathrm{kOe}$ for the same cooling field. Furthermore, it was found that the exchange bias in both cases increases with decreasing temperature of the sample. In Fig. 6 we see the dependence of $H_{\mathrm{ex}}$ on temperature of the sample that was observed to be stronger in the case of $20 \mathrm{kOe}$ as compared to $90 \mathrm{kOe}$ especially at low temperatures. A sharp rise in $H_{\mathrm{ex}}$ was observed at low temperatures like it was observed and discussed in the case of coercivity versus temperature curve (see Fig. 4) for the same sample. Because of the exchange coupling between the surface and the core spins, the field cooling can select a surface spin configuration which favors the particle being magnetized in the field cooling direction resulting in large coercivities and large exchange bias values. To explore the temperature dependence of exchange bias for our nanoparticles in detail, we refer to Fig. 6 where we see a sharp decrease in $H_{\text {ex }}$ with increasing temperature, starting from lower temperature side, and then the decrease is lower in the high temperature range of 50-250 K, while it vanishes beyond $250 \mathrm{~K}$. The decrease in $H_{\mathrm{ex}}$ with increasing temperature may be explained on the basis of the core-shell model in cobalt ferrite nanoparticles. The frozen disordered spin structure at low temperature $(10 \mathrm{~K})$ starts unfreezing with increasing temperature of the sample, thereby decreasing the surface anisotropy that is responsible for the exchange bias effects in these nanoparticles. Therefore the exchange bias decreases with increasing temperature of the sample. It has been reported in literature that the exchange bias generally disappears at a temperature below the blocking temperature of the FM/FI core $^{24}$ and that is $\sim 315 \mathrm{~K}$ for our nanoparticles as discussed earlier; we see that the value of $H_{\mathrm{ex}}$ is negligible even well below $T_{B}$. The large $H_{\mathrm{ex}}$ values at lower temperatures could also be due to the pronounced growth of magnetic anisotropy of the core and shell in $\mathrm{CoFe}_{2} \mathrm{O}_{4}$ nanoparticles. This growth of anisotropy resultantly increases the core-shell (spin) interaction that, in turn, increases the $H_{\mathrm{ex}}$ values at the lower temperatures.

When we are dealing with nanoparticles at low temperatures, the anisotropy may be a huge function of temperature. Thus other factors, apart from the enhancement of anisotropy, such as the structural properties that are intrinsic to the nanoparticles, including volume distribution, randomness of anisotropy axes, and interparticle interactions may also influence the thermal dependence of exchange bias and coercivity in nanoparticles. ${ }^{25}$ It is interesting to note that the exchange bias values are lower for the loops exposed to higher fields $( \pm 90 \mathrm{kOe})$ as compared to those taken at lower fields $( \pm 20 \mathrm{kOe})$. We attribute this to the high field effects where most of the surface spins turn along the field direction resulting in the reduction in the core-shell interface interaction and hence the exchange bias in core-shell cobalt ferrite nanoparticles.

\section{CONCLUSION}

Coercivity and saturation magnetization of $26 \pm 4 \mathrm{~nm}$ $\mathrm{CoFe}_{2} \mathrm{O}_{4}$ nanoparticles showed an increasing behavior with decreasing temperature. The enhanced coercivity at lower temperatures has been attributed to the increasing effects of thermal fluctuations of the blocked moments across the anisotropy barriers according to Kneller's law. The saturation magnetization of the nanoparticles followed the modified Bloch's law based on the modified spin- wave spectrum for nanoparticles due to their finite size effects. The exchange fields were found to increase with decreasing temperature of the sample in the range of 10-300 K. A sharp increase in the exchange bias values was found for the low applied fields, while a relatively low increase was found for the higher applied fields. This has been attributed to the high field effects where most of the surface spins turn along the field direction, leading to the weaker core-shell interface interactions that decreases the exchange bias effects in these nanoparticles.

\section{ACKNOWLEDGMENTS}

The authors acknowledge the Higher Education Commission (HEC) of Pakistan for providing the financial support for this research work. K. Maaz acknowledges Professor J. Liu of the Institute of Modern Physics, Chinese Academy of Sciences, Lanzhou, P.R. China, for her financial support through the National NSF of China (Grant Nos. 10775161, 10775162, and 10805062), and the West Light Foundation of Chinese Academy of Sciences projects.

${ }^{1}$ P. Tartaj, M. D. Morales, and V. V. Sabino, J. Phys. D 36, R182 (2003).

${ }^{2}$ S. D. Bader, Rev. Mod. Phys. 78, 1 (2006).

${ }^{3}$ C. A. Ross, Annu. Rev. Mater. Res. 31, 203 (2001).

${ }^{4}$ R. W. Wood, J. Miles, and T. Olson, IEEE Trans. Magn. 38, 1711 (2002).

${ }^{5}$ C. J. Brumlik and C. R. Martin, Anal. Chem. 59, 2625 (1992).

${ }^{6}$ Z. Cai and C. R. Martin, J. Am. Chem. Soc. 111, 4138 (1989).

${ }^{7}$ S. K. Chakarvarti and J. Vetter, Nucl. Instrum. Methods Phys. Res. B 62, 109 (1991). 
${ }^{8}$ S. K. Chakarvarti and J. Vetter, J. Micromech. Microeng. 3, 57 (1993); Radiat. Meas. 29, 149 (1998).

${ }^{9}$ D. J. Craik, Magnetic Oxides (Wiley, London, 1975), pt. 2, p. 703.

${ }^{10}$ L. D. Tung, V. Kolesnichenko, D. Caruntu, N. H. Chou, C. J. O'Connor, and L. Spinu, J. Appl. Phys. 93, 7486 (2003).

${ }^{11}$ S. T. Alone and K. M. Jadhav, Pramana, J. Phys. 70, 173 (2008).

${ }^{12}$ R. H. Kodama and A. E. Berkowitz, J. Appl. Phys. 81, 5552 (1997).

${ }^{13}$ R. H. Kodama and A. E. Berkowitz, Phys. Rev. Lett. 77, 394 (1996).

${ }^{14}$ K. Maaz, A. Mumtaz, S. K. Hasanain, and A. Ceylan, J. Magn. Magn. Mater. 308, 289 (2007).

${ }^{15}$ K. Maaz, S. Karim, A. Mumtaz, S. K. Hasanain, J. Liu, and J. L. Duan, J. Magn. Magn. Mater. 321, 1838 (2009).

${ }^{16}$ JCPDS Card No. 791744.
${ }^{17}$ T. Fukui, C. Sakurai, and M. Okuyama, J. Mater. Res. 7, 791 (1992).

${ }^{18}$ E. F. Kneller and F. E. Luborsky, J. Appl. Phys. 34, 656 (1963).

${ }^{19}$ X. Batlle, M. Garcia del Muro, J. Tejada, H. Pfeiffer, P. Goand, and E. Sinn, J. Appl. Phys. 74, 3333 (1993).

${ }^{20}$ O. Iglesias and A. Labarta, Phys. Rev. B 63, 184416 (2001).

${ }^{21}$ F. Bloch, Z. Phys. 61, 206 (1931).

${ }^{22}$ K. Mandal, S. Mitra, and P. Anil Kumar, Europhys. Lett. 75, 618 (2006).

${ }^{23}$ A. Auino, M. H. Sousa, H. R. Rechenbrg, G. F. Goya, F. A. Tourinho, and J. Depeyrot, J. Metastable Nanocryst. Mater. 20-21, 694 (2004).

${ }^{24}$ F. Radu, M. Etzkorn, R. Schmitte, K. Westerholt, and H. Zabel, Phys. Rev. B 67, 134409 (2003).

${ }^{25}$ O. Iglesias, A. Labarta, and X. Batlle, J. Nanosci. Nanotechnol. 8, 2761 (2008). 